\title{
BOUNDS FOR CLASSICAL POLYNOMIALS DERIVABLE BY MATRIX METHODS
}

\section{J. L. BRENNER ${ }^{1}$}

ABSTRACT. For a matrix with dominant diagonal, convenient upper and lower bounds for the determinant are available. Such bounds occur in the work of Price, Ostrowski, Hoffman, Haynsworth, and Brenner. In this article, the inequalities are used to bound the classical polynomials over a range of values of the argument.

1. Introduction. Let $A$ be a square matrix of complex numbers. Bounds on $\operatorname{det} A$ are known (see below) when the elements $a_{i j}$ satisfy the hypotheses in Definitions 1.01, 1.03.

1.01. Definition. $A=\left[a_{i j}\right]$ is said to have (strictly) dominant diagonal if

(1.02) $\forall_{k}\left\{\left|a_{k k}\right|>\sum_{i \neq k}\left|a_{k i}\right|=R_{k}\right\}$, i.e. the diagonal element dominates in each row.

1.03. Definition. If a matrix has dominant diagonal, $\forall_{k}\left\{a_{k k} \neq 0\right\}$, the numbers $\sigma_{k}$ are defined by

(1.04) $\sigma_{k}\left|a_{k k}\right|=R_{k}$.

Thus $0 \leqq \sigma_{k}<1$.

1.05. TheOREM [4]. Let $A$ be an $n \times n$ matrix of complex numbers and let (1.02) hold. Then

(1.06) $\prod L_{k} \leqq|\operatorname{det} A| \leqq \prod U_{k}$,

where $L_{k}=\left|a_{k k}\right|-\sum_{j>k}\left|a_{k j}\right|, \quad U_{k}=\left|a_{k k}\right|+\sum_{j>k}\left|a_{k j}\right|, \quad k=1(1) n$, $L_{n}=U_{n}=\left|a_{n n}\right|$.

1.07. TheOREM [1]. Let $A$ be an $n \times n$ matrix of complex numbers and let (1.04) hold. Then

(1.08) $\prod L_{k}{ }^{\prime} \leqq|\operatorname{det} A| \leqq \prod U_{k}{ }^{\prime}$, where $L_{k}^{\prime}=\left|a_{k k}\right|-\sum_{j>k} \sigma_{j}\left|a_{k j}\right|, U_{k}{ }^{\prime}=\left|a_{k k}\right|+\sum_{j>k} \sigma_{j}\left|a_{k j}\right|$.

\section{Special inequalities.}

2.01. First consider the $n \times n$ matrix $A_{n}=[-1-2 \lambda] I-\lambda\left(K+K^{*}\right)$, where $K$ is the matrix with 1 's on the superdiagonal and zeros elsewhere. $A_{n}$ was used by Crank and Nicolson [2] to solve the heat

Presented to the Society, April 24, 1971; received by the editors November 30, 1970.

AMS 1970 subject classifications. Primary 33A65; Secondary 15A15.

Key words and phrases. Orthogonal polynomials.

1 Supported by NSF GP-9483. 
equation numerically. Let $D_{n}=\operatorname{det} A_{n}$. The three-term recursion relation

(2.02) $D_{n}=(-1-2 \lambda) D_{n-1}-\lambda^{2} D_{n-2}$ is verified for $n=1,2, \cdots$, with the conventions $D_{0}=1, D_{-1}=0$.

Using (2.02), $D_{n}$ is easily evaluated as

(2.03) $D_{n}=\left(\alpha_{1}^{n+1}-\alpha_{2}^{n+1}\right) /\left(\alpha_{1}-\alpha_{2}\right)$,

where $\alpha_{1}, \alpha_{2}$ are the roots of the indicial equation for (2.02): $\alpha_{1,2}=\frac{1}{2}\left\{-1-2 \lambda+(1+4 \lambda)^{1 / 2}\right\}$.

On the other hand, the matrix $A_{n}$ has dominant diagonal (satisfies (1.02)) if $\operatorname{Re} \lambda>-\frac{1}{4}$. Thus for all values of $\lambda$ such that $\operatorname{Re} \lambda>-\frac{1}{4}$, application of Theorem 1.5 leads to the following corollary inequalities.

$$
\begin{aligned}
& |1+2 \lambda|\{|1+2 \lambda|-|\lambda|\}^{n-1} \\
& \leqq \mid 2^{-n-1}\left[-1-2 \lambda+(1+4 \lambda)^{1 / 2}\right]^{n+1} /(1+4 \lambda)^{1 / 2} \\
& -2^{-n-1}\left[-1-2 \lambda-(1+4 \lambda)^{1 / 2}\right]^{n+1} /(1+4 \lambda)^{1 / 2} \mid \\
& \leqq|1+2 \lambda|\{|1+2 \lambda|+|\lambda|\}^{n-1} \text {. }
\end{aligned}
$$

In particular if $\lambda$ is real and positive, (2.04) simplifies to

$$
\begin{aligned}
2^{n+1}(1 & +2 \lambda)(1+\lambda)^{n-1}(1+4 \lambda)^{1 / 2} \\
& <\left(1+2 \lambda+(1+4 \lambda)^{1 / 2}\right)^{n+1}-\left(1+2 \lambda-(1+4 \lambda)^{1 / 2}\right)^{n+1} \\
& <2^{n+1}(1+2 \lambda)(1+3 \lambda)^{n-1}(1+4 \lambda)^{1 / 2}
\end{aligned}
$$

For $\lambda$ in the interval $\left(-\frac{1}{4}, 0\right)$, the inequality signs in (2.05) are reversed.

2.06. A matrix similar to $A_{n}$. Although the $n \times n$ matrix $B_{n}$ $=\left[\alpha_{1}+\alpha_{2}\right] I+\alpha_{2} K+\alpha_{1} K^{*}$ does not have dominant diagonal, nevertheless if $\arg \alpha_{1}=\arg \alpha_{2}$, the conclusions of Theorems 1.05, 1.07 do apply to this matrix, as a continuity argument shows. Actually, $B_{n}$ is similar to $A_{n}: S B_{n} S^{-1}=A_{n}$, where $S=\operatorname{diag}\left[d_{11}, d_{22}, \cdots, d_{n n}\right]$, with $d_{j j}=\left(\alpha_{2} / \alpha_{1}^{j}\right)^{1 / 2}$.

The bounds obtainable from this matrix are less interesting; they amount to the following:

2.07. ThEOREM. If $\alpha_{1}>\alpha_{2}>0$, then (see (2.03))

$$
\alpha_{1}^{n-1}\left(\alpha_{1}^{2}-\alpha_{2}^{2}\right)<\alpha_{1}^{n+1}-\alpha_{2}^{n+1}<\left(\alpha_{1}+2 \alpha_{2}\right)^{n-1}\left(\alpha_{1}^{2}-\alpha_{2}^{2}\right) .
$$

2.08. Another matrix similar to $A_{n}$. If more conditions are imposed on $\alpha_{1}, \alpha_{2}$, a more interesting inequality will arise. Consider the $n \times n$ matrix $C_{n}=\left(\alpha_{1}+\alpha_{2}\right) I+\alpha_{1} \alpha_{2} K+K^{*}$. This matrix has $\alpha_{1}+\alpha_{2}$ on the diagonal, $\alpha_{1} \alpha_{2}$ on the superdiagonal, and 1 on the subdiagonal. The 
relation $T C_{n} T^{-1}=B_{n}$, where $T=\operatorname{diag}\left[\alpha_{1}, \alpha_{1}^{2}, \cdots, \alpha_{1}^{n}\right]$ shows that $C_{n}$ is similar to $B_{n}$ (and hence to $A_{n}$ ). Application of Theorem 1.05 yields

2.09. THEOREM. Let $\alpha_{1}, \alpha_{2}$ be complex numbers such that $\left|\alpha_{1}+\alpha_{2}\right|$ $>1+\left|\alpha_{1} \alpha_{2}\right|$. Then

$$
\begin{aligned}
& \left\{\left|\alpha_{1}+\alpha_{2}\right|-\left|\alpha_{1} \alpha_{2}\right|\right\}^{n-1}\left|\alpha_{1}^{2}-\alpha_{2}^{2}\right| \\
& \quad \leqq\left|\alpha_{1}^{n+1}-\alpha_{2}^{n+1}\right| \leqq\left\{\left|\alpha_{1}+\alpha_{2}\right|+\left|\alpha_{1} \alpha_{2}\right|\right\}^{n-1}\left|\alpha_{1}^{2}-\alpha_{2}^{2}\right| .
\end{aligned}
$$

This result might be tedious to verify otherwise, especially when $\alpha_{1}, \alpha_{2}$ are not real.

2.11. A generalization. The matrix $E_{n}=T_{1} B_{n} T_{1}^{-1}$, where $T_{1}$ $=\operatorname{diag}\left[1, k_{2}, k_{2} k_{3}, \cdots, k_{2} k_{3} \cdots k_{n}\right]$, leads to the following result.

2.12. TheOREM. Let $\alpha_{1}, \alpha_{2}, k_{2}, k_{3}, \cdots, k_{n}$ be complex numbers such that the $n$ inequalities $\left|\alpha_{1}+\alpha_{2}\right|>\left|\alpha_{1} / k_{j}\right|+\left|k_{j+1} \alpha_{2}\right|$ hold for $j=1(1) n$ $\left(k_{1}=\infty, k_{n+1}=0\right)$. Then

$$
\begin{aligned}
\left|\alpha_{1}^{2}-\alpha_{2}^{2}\right| \prod_{j=2}^{n}\left\{\left|\alpha_{1}+\alpha_{2}\right|-\left|k_{j} \alpha_{2}\right|\right\} \\
\quad \leqq\left|\alpha_{1}^{n+1}-\alpha_{2}^{n+1}\right| \leqq\left|\alpha_{1}^{2}-\alpha_{2}^{2}\right| \prod_{j=2}^{n}\left\{\left|\alpha_{1}+\alpha_{2}\right|+\left|k_{j} \alpha_{2}\right|\right\}
\end{aligned}
$$

Again these relations appear unpleasant to establish directly, especially so in case some of the variables are not real.

2.13. Arbitrary diagonal elements. To obtain the bounds of this section directly would not be difficult. But with absolutely no effort, Theorem 2.14 falls out as a corollary of Theorems 1.05 and 1.07.

2.14. ThEOREM. Let $a(n)$ be a complex-valued function of $n$, and let $\lambda$ be a complex parameter such that

$|a(1)|>|\lambda|, \quad|a(j)|>|2 \lambda| \quad(j=2(1) n-1),|a(n)|>|\lambda|$.

Let the numbers $E_{n}$ be defined by the recursion

$$
E_{-1}=0, \quad E_{0}=1, \quad E_{j}=a(j) E_{j-1}-\lambda^{2} E_{j-2}, \quad j=1(1) n .
$$

Then the inequalities

$$
|a(1)| \prod_{j=2}^{n}(|a(j)|-|\lambda|) \leqq\left|E_{n}\right| \leqq|a(1)| \prod_{j=2}^{n}(|a(j)|+|\lambda|)
$$

are valid bounds on $\left|E_{n}\right|$.

Application of Theorem 1.07 would yield more precise bounds for 
$\left|E_{n}\right|$. Indeed, $E_{n}=\operatorname{det}\left\{\operatorname{diag}[a(n), \cdots, a(1)]+\lambda\left[K+K^{*}\right]\right\}$. Note that $E_{1}=a(1) ; E_{2}=a(2) a(1)-\lambda^{2}$.

3. A special polynomial. The formula

$$
\sin (n+1) \theta / \sin \theta=\operatorname{det}\left\{2 \cos \theta I+K+K^{*}\right\}
$$

is derivable (by induction) from a simple trigonometric identity. Furthermore it is possible to establish the relation

$$
\begin{aligned}
& \sin (n+1) \theta / \sin \theta \\
& =(2 \cos \theta)^{n}-\left(\begin{array}{c}
n-1 \\
1
\end{array}\right)(2 \cos \theta)^{n-2}+\left(\begin{array}{c}
n-2 \\
2
\end{array}\right)(2 \cos \theta)^{n-4}-\cdots \\
& \quad+(-1)^{p}\left(\begin{array}{c}
n-p \\
p
\end{array}\right)(2 \cos \theta)^{n-2 p}+\cdots
\end{aligned}
$$

see [3, Problems 52,53]. The algebraic identity

$$
\operatorname{det}\left\{x I+K+K^{*}\right\}=\sum_{p=0}^{[n / 2]}(-1)^{p}\left(\begin{array}{c}
n-p \\
p
\end{array}\right) x^{n-2 p}
$$

follows. If $|x| \geqq 2$, the matrix $x I+K+K^{*}$ has dominant diagonal. From this fact, Theorem 3.04 is a corollary.

3.04. Theorem. Let $x$ be a complex number, $|x| \geqq 2$. Then the inequalities

$$
\begin{aligned}
|x| \cdot(|x|-1)^{n-1} & \leqq\left|\sum_{p=0}^{[n / 2]}(-1)^{p}\left(\begin{array}{c}
n-p \\
p
\end{array}\right) x^{n-2 p}\right| \\
& \leqq|x| \cdot(|x|+1)^{n-1}
\end{aligned}
$$

hold if $n \geqq 2$.

Further, from Theorem 1.07, one obtains:

3.05. THEOREM. If $x \geqq 2, n \geqq 2$ then the inequalities

$$
\begin{aligned}
\left(|x|^{2}-1\right)\left(|x|^{2}-2\right)^{n-2} / \mid & \left.x\right|^{n-2} \\
& \leqq\left|\sum_{p=0}^{[n / 2]}(-1)^{p}\left(\begin{array}{c}
n-p \\
p
\end{array}\right) x^{n-2 p}\right| \\
& \leqq\left(|x|^{2}+1\right)\left(|x|^{2}+2\right)^{n-2} /|x|^{n-2}
\end{aligned}
$$

hold. 
The polynomial being estimated in (3.06) is the polynomial $\sin [(n+1) \operatorname{arc} \cos (x / 2)] / \sin \arccos (x / 2)$.

\section{4. Čebyšev polynomials.}

4.01. Lemma. The Čebyร̌ev polynomial $T_{n}(x)=\cos (n \operatorname{arc} \cos x)$ satisfies the three-term recursion relation $T_{n}(x)=2 x T_{n-1}(x)-T_{n-2}(x)$, $n \geqq 1$, with $T_{0}(x) \equiv 1, T_{-1}(x) \equiv 0$.

4.02. Lemma. $T_{n}(x)$ is given by the formula

$$
T_{n}(x)=\operatorname{det}\left\{K+K^{*}+\operatorname{diag}[2 x, \cdots, 2 x, x]\right\} .
$$

Proof. The determinant has the correct starting values and satisfies the correct three-term recursion.

From Theorems 1.05, 1.07 the estimates below are obtained.

4.03. ThEOREM. Let $|x| \geqq 1, n \geqq 1$. Then

$$
[2|x|-1]^{n-1}|x| \leqq\left|T_{n}(x)\right| \leqq[2|x|+1]^{n-1}|x| \text {. }
$$

4.05. ThEOREM. Let $|x| \geqq 1, n \geqq 2$. Then

$$
\begin{aligned}
\left(2|x|^{2}-1\right)^{n-1} /|x|^{n-2} & \leqq\left|T_{n}(x)\right| \\
& \leqq\left(2|x|^{2}+1\right)^{n-1} /|x|^{n-2} .
\end{aligned}
$$

Estimates for $\left|T_{n}(x)\right|$ are well known when $x$ is real, $|x| \leqq 1$. The estimates given by Theorems 4.04, 4.06 are reasonable; they grow about as fast as a polynomial of degree $n$, which $T_{n}(x)$ certainly is: $T_{n}(x)=2^{n-1} x^{n}+\cdots$.

5. Hermite polynomials. Let $H_{n}=(-1)^{n} e^{x^{2} / 2} D^{n} e^{-x^{2} / 2}$ be the $n$th Hermite polynomial, so that $H_{0}=1, H_{1}=x, H_{2}=x^{2}-1, H_{3}=x^{3}-3 x$, $H_{4}=x^{4}-6 x^{2}+3, H_{5}=x^{5}-10 x^{3}+15 x$. Then

(5.01) $H_{n+1}(x)=x H_{n}(x)-n H_{n-1}(x)$.

Therefore

(5.02) $H_{n}(x)=\operatorname{det}\left[x I+K^{*}+W_{4}\right]$, where $W_{4}$ has superdiagonal $n-1, n-2, \cdots, 1$ and 0 's elsewhere. This gives the following bounds.

5.03. THEOREM. For $|x|>n>0$, the Hermite polynomial $H_{n}(x)$ $=(-1)^{n} e^{x^{2} / 2} D^{n} e^{-x^{2} / 2}$ satisfies the following inequalities:

$$
\prod_{j=0}^{n-1}(|x|-j) \leqq\left|H_{n}(x)\right| \leqq \prod_{j=0}^{n-1}(|x|+j),
$$




$$
\begin{aligned}
\left(|x|^{2}-n+1\right) & \prod_{j=1}^{n-1}\left(|x|^{2}-j\right) \\
& \leqq|x|^{n}\left|H_{n}(x)\right| \leqq\left(|x|^{2}+n-1\right) \prod_{j=1}^{n-1}\left(|x|^{2}+j\right)
\end{aligned}
$$

Bounds for a larger range of values of $x$ are obtained by using the matrix $M=x I+W_{5}+W_{5}^{*}$, where each element of $W_{5}$ is the square root of the corresponding element of $W_{4}$; this matrix is similar to the preceding one.

5.06. THEOREM. Let $|x|>(n-1)^{1 / 2}+(n-2)^{1 / 2}, n>1$. Then $H_{n}(x)$ $=(-1)^{n} e^{x^{2} / 2} D^{n} e^{-x^{2} / 2}$ is bounded as follows.

$$
\begin{aligned}
& \prod_{j=0}^{n-1}(|x|-\sqrt{ } j) \leqq\left|H_{n}(x)\right| \leqq \prod_{j=0}^{n-1}(|x|+\sqrt{ } j) \\
& \left(|x|^{2}-1\right) \prod_{j=2}^{n-1}\left(|x|^{2}-2 j\right) /|x|^{n-2} \\
& \leqq\left|H_{n}(x)\right| \leqq\left(|x|^{2}+1\right) \prod_{j=2}^{n-1}\left(|x|^{2}+2 j\right) /|x|^{n-2}
\end{aligned}
$$

5.09. REMARK. Note that these bounds are in general better than the bounds of the preceding theorem, but they are invalid for $n=1$.

5.10. The recursion formula for

$$
K_{n}(x)=n H_{n}(x)=(-1)^{n} n e^{x^{2} / 2} D^{n} e^{-x^{2} / 2}
$$

is

$$
K_{n+1}(x)=x\left(1+\frac{1}{n}\right) K_{n}(x)-\left(n+2+\frac{2}{n-1}\right) K_{n-1}(x),
$$

which is asymptotically like the recursion formula (5.01) for $H_{n}(x)$. This gives bounds for $K_{n}(x)$, and hence for $H_{n}(x)$, that are better than the preceding bounds, for some moderately large values of $x$. Since the bounds are, for very large $x$, inferior to the preceding, the details are suppressed.

6. Legendre polynomials. The polynomials

$$
M_{n}(x)=2^{-n}(n !)^{-1} D^{n}\left(x^{2}-1\right)^{n}
$$

satisfy the recursion $(n>0)$

$$
\begin{aligned}
n M_{n}(x)=(2 n-1) x M_{n-1}(x)-(n-1) M_{n-2}(x), & \\
M_{0}=1, M_{-1} & =0
\end{aligned}
$$


Therefore $M_{0}=1, \quad M_{1}=x, \quad M_{2}=\frac{1}{2}\left(3 x^{2}-1\right), \quad M_{3}=\frac{1}{2}\left(5 x^{3}-3 x\right), \quad M_{4}=$ $\frac{1}{8}\left(35 x^{4}-30 x^{2}+3\right) ; M_{n}(x)=\operatorname{det}\left\{x W_{6}+W_{7}+W_{7}^{*}\right\}$, where

$$
\begin{aligned}
& W_{6}=\operatorname{diag}\left[2-n^{-1}, 2-(n-1)^{-1}, \cdots, 2-1\right], \\
& W_{7}=\text { super } \operatorname{diag}\left[1-n^{-1}, \cdots, 1-2^{-1}\right] .
\end{aligned}
$$

6.02. Theorem. Let $n \geqq 2$. Then $M_{n}(x)=2^{-n}(n !)^{-1} D^{n}\left(x^{2}-1\right)^{n}$ is bounded as follows for $|x| \geqq 4 / 3$.

$$
\begin{aligned}
|x| \prod_{j=2}^{n} & \left\{\left(2-j^{-1}\right)|x|-\left(1-j^{-1}\right)^{1 / 2}\right\} \\
& \leqq\left|M_{n}(x)\right| \leqq|x| \prod_{j=2}^{n}\left\{\left(2-j^{-1}\right)|x|+\left(1-j^{-1}\right)^{1 / 2}\right\} .
\end{aligned}
$$

The range of validity of these inequalities shrinks down towards $|x|>1$ for sufficiently large $n$.

7. Laguerre polynomials. The Laguerre polynomials are defined by

$$
\begin{aligned}
L_{n}(x) & =(-1)^{n} e^{x} \frac{d^{n}}{d x^{n}} x^{n} e^{-x} \\
& =x^{n}-n^{2} x^{n-1}+\frac{n^{2}(n-1)^{2}}{2 !} x^{n-2} \cdots \pm n !
\end{aligned}
$$

They satisfy the recursion relation $L_{n+1}=(x-2 n-1) L_{n}-n^{2} L_{n-1}$ $(n \geqq 0) ; L_{0}=1$.

Therefore $L_{n}(n>1)$ is given by $L_{n}(x)=\operatorname{det}\left\{W_{8}+W_{9}+W_{9}^{*}\right\}$, where

$$
\begin{aligned}
& W_{8}=\operatorname{diag}[x-2 n+1, x-2 n+3, \cdots, x-3, x-1], \\
& W_{9}=\operatorname{super} \operatorname{diag}[n-1, n-2, \cdots, 2,1] .
\end{aligned}
$$

This leads to the following estimates.

7.02. THEOREM. For $n>3$, if $|x-2 n+3| \geqq 2 n-3$, then $L_{n}(x)$ $=(-1)^{n} e^{x} D^{n}\left(x^{n} e^{-x}\right)$ satisfies

$$
\begin{aligned}
|x-1| \prod_{j=1}^{n-1} & \{|x-2 j-1|-j\} \\
& \leqq\left|L_{n}(x)\right| \leqq|x-1| \prod_{j=1}^{n-1}\{|x-2 j-1|+j\} .
\end{aligned}
$$

7.04. THEOREM. For $n>3$, if $|x-2 n+3| \geqq 2 n-3$, then $L_{n}(x)$ $=(-1)^{n} e^{x} D^{n}\left(x^{n} e^{-x}\right)$ satisfies 


$$
\begin{aligned}
\left|x^{2}-4 x+2\right| & \prod_{j=2}^{n}\left|x^{2}-4 j x+2 j^{2}+j-1\right| \\
& \leqq\left|L_{n}(x)\right| \cdot \prod_{j=2}^{n-1}|x-2 j+1| \\
& \leqq\left|x^{2}-4 x+4\right| \prod_{j=2}^{n}\left|x^{2}-4 j x+6 j^{2}-j-1\right|
\end{aligned}
$$

In particular, these inequalities are valid for all nonpositive $x$ $(x \leqq 0)$. The lower bound for $\left|L_{n}(0)\right|$ is exact.

8. Generalized Laguerre polynomials. The modified Laguerre polynomial is

$$
\begin{gathered}
L_{n}^{a}(x)=n !^{-1} e^{x} x^{-a} D^{n}\left(e^{-x} x^{n+a}\right) ; \\
L_{-1}^{a}=0, \quad L_{0}^{a}=1, \quad L_{1}^{a}=-x+1+a, \\
L_{2}^{a}=x^{2}-2(2+a) x+(2+a)(1+a), \cdots .
\end{gathered}
$$

These polynomials satisfy the recursion

$$
(n+1) L_{n+1}^{a}=(2 n+a+1-x) L_{n}^{a}-(n+a) L_{n-1}^{a}, \quad n \geqq 0 .
$$

In (8.02), (8.03), $a$ can be any complex number. In Theorem 8.05, $a$ is real. Thus $L_{n}^{a}(x)=\operatorname{det}\left\{W_{10}+W_{11}+W_{11}^{*}\right\}$, where

$$
W_{10}=\operatorname{diag}\left[n^{-1}(2 n+a-1-x),(n-1)^{-1}(2 n+a-3-x),\right.
$$

$$
\left.\cdots, 2^{-1}(a+3-x), a+1-x\right]
$$

$$
\begin{array}{r}
W_{11}=\operatorname{super} \operatorname{diag}\left[n^{-1 / 2}(n+a-1)^{1 / 2},(n-1)^{-1 / 2}(n+a-2)^{1 / 2}\right. \\
\left.\cdots, 2^{-1 / 2}(a+1)^{1 / 2}\right] .
\end{array}
$$

If $a \geqq 4 / 3, n \geqq 4$, the diagonal of this matrix dominates for all $x$ such that $|2 n+a-3-x| \geqq 2((n-1)(n+a-2))^{1 / 2}$.

8.05. ThEOREM. Let $a \geqq 4 / 3, n \geqq 4$. For all $x$ such that $|2 n+a-3-x|$ $\geqq 2((n-1)(n+a-2))^{1 / 2}$, the modified Laguerre polynomial $L_{n}^{a}(x)$ $=n !^{-1} e^{x} x^{-a} D^{n}\left(e^{-x} x^{n+a}\right)$ satisfies

$$
\begin{aligned}
\mid a+1 & -x\left|\prod_{j=2}^{n}\left\{|a-1-x+2 j|-((j+a-1) / j)^{1 / 2}\right\} \leqq\right| L_{n}^{a}(x) \mid \\
& \leqq|a+1-x| \prod_{j=2}^{n}\left\{|a-1-x+2 j|+((j+a-1) / j)^{1 / 2}\right\} .
\end{aligned}
$$


9. Arctangent polynomials. The polynomials $S_{n}(x)$ defined by

$$
n ! S_{n}(x)=(-1)^{n}\left(1+x^{2}\right)^{n+1} D^{n}\left(1+x^{2}\right)^{-1}
$$

satisfy the recursion $(n>0)$ [3]

$$
S_{n}=2 x S_{n-1}-\left(x^{2}+1\right) S_{n-2}, \quad S_{-1}=0, S_{0}=1 .
$$

For a small range of values of $\operatorname{Re} x, \operatorname{Im} x$, bounds for these polynomials can be obtained. The $n \times n$ matrix $(n>0) \quad W_{12}=2 \times I+$ $\left(x^{2}+1\right)^{1 / 2} K$ has dominant diagonal if $|x| \geqq\left|\left(x^{2}+1\right)^{1 / 2}\right|$, i.e. if $2[\operatorname{Im} x]^{2} \geqq\left(1+2[\operatorname{Re} x]^{2}\right) /\left(1-[\operatorname{Re} x]^{2}\right)$.

9.03. Theorem. For $n>0,\left|x^{2}\right| \geqq\left|x^{2}+1\right|$, the polynomial $S_{n}(x)$ $=(-1)^{n}(n !)^{-1}\left(1+x^{2}\right)^{n+1} D^{n}\left(1+x^{2}\right)^{-1}$ satisfies the relations:

$$
\begin{aligned}
& |2 x|\left(|2 x|-\left|\left(x^{2}+1\right)^{1 / 2}\right|\right)^{n-1} \\
& \quad \leqq\left|S_{n}(x)\right| \leqq|2 x|\left(|2 x|+\left(x^{2}+1\right)^{1 / 2}\right)^{n-1} ; \\
& 2\left\{\left|2 x^{2}\right|-\left|x^{2}+1\right|\right\}^{n-2}\left\{\left|4 x^{2}\right|-\left|x^{2}+1\right|\right\} \\
& \quad \leqq\left|x^{n-2} S_{n}(x)\right| \leqq 2\left\{\left|2 x^{2}\right|+\left|x^{2}+1\right|\right\}^{n-2}\left\{\left|4 x^{2}\right|+\left|x^{2}+1\right|\right\} .
\end{aligned}
$$

9.04. Arcsinh polynomials. The polynomials

$$
U_{n}(x)=(-1)^{n}\left(1+x^{2}\right)^{n+1 / 2} D^{n}\left(1+x^{2}\right)^{-1 / 2}
$$

satisfy the recursion $(n>0)$

$$
\begin{aligned}
U_{n}=(2 n-1) x U_{n-1}-(n-1)^{2}(1+x)^{2} U_{n-2}, & \\
& U_{0}=1, U_{-1}=0 .
\end{aligned}
$$

Therefore $U_{n}(x)=\operatorname{det}\left\{W_{14}+W_{13}+W_{13}^{*}\right\}$, where

$$
\begin{aligned}
& W_{14}=x \operatorname{diag}[2 n-1,2 n-3, \cdots, 3,1] ; \\
& W_{13}=\left(1+x^{2}\right)^{1 / 2} W_{9} .
\end{aligned}
$$

9.08. TheOREM. For $n>0,\left|x^{2}\right| \geqq\left|x^{2}+1\right|$, the polynomial $U_{n}(x)$ $=(-1)^{n}\left(1+x^{2}\right)^{n+1 / 2} D^{n}\left(1+x^{2}\right)^{1 / 2}$ satisfies the inequalities

$$
\begin{aligned}
& |x|\left\{(2 n-1)|x|-(n-1)\left|\left(1+x^{2}\right)^{1 / 2}\right|\right\}\left\{|x|-\left|\left(1+x^{2}\right)^{1 / 2}\right|\right\}^{n-2} \\
& \leqq\left|U_{n}(x)\right| / \prod_{j=1}^{n-2}(2 j+1) \\
& \leqq|x|\left\{(2 n-1)|x|+(n-1)\left|\left(1+x^{2}\right)^{1 / 2}\right|\right\} \\
& \cdot\left\{|x|+\left|\left(1+x^{2}\right)^{1 / 2}\right|\right\}^{n-2} \text {. }
\end{aligned}
$$

9.10. Polynomials derived from $\exp (1 / x)$. The polynomials $V_{n}$ defined by (see [5]) 


$$
V_{n}(x)=(-1)^{n+1} x^{2 n+2} \exp (-1 / x) D^{n+1} \exp (1 / x)
$$

satisfy the recursion $(n>0)$

$$
V_{n}=(2 n x+1) V_{n-1}-n(n-1) x^{2} V_{n-2} .
$$

Therefore $V_{n}(x)=\operatorname{det}\left\{I+2 x W_{16}+x W_{15}+x W_{15}^{*}\right\}$, where $W_{16}=$ $\operatorname{diag}[n, n-1, \cdots, 1], W_{15}=\operatorname{super} \operatorname{diag}[n, n-1, \cdots, 2]$. For $n>1$, this matrix has dominant diagonal if $\operatorname{Re} x \geqq-1 /(4 n-4)$, in particular if $\operatorname{Re} x \geqq 0$. Bounds are easily derived; details are suppressed.

\section{REFERENCES}

1. J. L. Brenner, $A$ bound for a determinant with dominant main diagonal, Proc. Amer. Math. Soc. 5 (1954), 631-634. MR 16, 105.

2. J. Crank and P. Nicolson, A practical method for numerical evaluation of solutions of partial differential equations of the heat-conduction type, Proc. Cambridge Philos. Soc. 43 (1947), 50-67. MR 8, 409.

3. D. K. Faddeev and I. S. Sominskir, Collection of problems on higher algebra, GITTL, Moscow, 1952; English transl., Problems in higher algebra, Freeman, San Francisco, Calif., 1965. MR 14, 1055; MR 31 \#1258.

4. G. B. Price, Bounds for determinants with dominant principal diagonal, Proc. Amer. Math. Soc. 2 (1951), 497-502. MR 12, 793.

5. I. Schur, Beispiele für Gleichungen ohne Affekt, Jber. Deutsch. Math.-Verein. 29 (1920).

University of Arizona, Tucson, Arizona 85721

University of Victoria, Victoria, British Columbia, Canada 